\title{
Airfoil optimization for noise emission problem and aerodynamic performance criterion on small scale wind turbines
}

\author{
Tuhfe Göçmen ${ }^{\mathrm{a}, *}$, Barış Özerdem ${ }^{\mathrm{a}, \mathrm{b}}$ \\ ${ }^{a}$ Department of Mechanical Engineering, İzmir Institute of Technology, Gülbahçe, 35430 Urla-Izmir, Turkey \\ ${ }^{\mathrm{b}}$ Department of Energy Systems Engineering, Bahcesehir University, 34353 Besiktas-Istanbul, Turkey
}

\section{A R T I C L E I N F O}

\section{Article history:}

Received 13 September 2011

Received in revised form

17 May 2012

Accepted 18 May 2012

Available online 18 June 2012

\section{Keywords:}

Small scale wind turbine

Noise emission

Aerodynamic performance

Aero-acoustics

Airfoil optimization

\begin{abstract}
A B S T R A C T
Noise emission is one of the major concerns in wind turbine industry and especially for small scale wind turbines, which are mostly erected to the urban areas; the concern is turning into a problem. This paper focuses on the optimization of six airfoils which are widely used on small scale wind turbines in terms of the noise emission and performance criteria and the numerical computations are performed for a typical $10 \mathrm{~kW}$ wind turbine. The main purpose of this optimization process is to decrease the noise emission levels while increasing the aerodynamic performance of a small scale wind turbine by adjusting the shape of the airfoil. The sources of the broadband noise emission are defined and their dominancy is investigated with respect to the operating conditions. While redesigning, together with the principals of reducing the airfoil self-noise, the aerodynamic prospects of increasing the performance have been taken into account. The codes which are based on aero-acoustic empirical models and a collection of wellknown aerodynamic functions are used in this study. The results obtained from the numerical analysis of the optimization process have shown that, the considered commercial airfoils for small scale wind turbines are improved in terms of aero-acoustics and aerodynamics. The pressure sides of the baseline airfoils have been manipulated together with the trailing edge and redesigned airfoils have lower levels of noise emission and higher lift to drag ratios.
\end{abstract}

(ㄷ) 2012 Elsevier Ltd. All rights reserved.

\section{Introduction}

The technological research on sustainable energy has been propagating and accelerating all around the world as a result of the energy politics gained today. In this context, the wind power is playing an important role contributing to this development. The small scale wind turbines seem to contribute a lot to this development, especially in Turkey, because of its potential and the recent legal arrangement done on this subject. However, the wind turbine noise is one of the major obstacles on the widespread use of wind power. The main sources of wind turbine noise may be divided into two: mechanical noise which includes the noise from the fans, generator, gear box etc., and the aerodynamic noise which is originated from the interaction between the rotor and the wind. Nowadays the mechanical noise is mostly handled; therefore the main engineering effort is being made on the subject of aerodynamic noise research [1]. There are five self-noise mechanisms on an airfoil, aerodynamically: Turbulent boundary layer trailing

\footnotetext{
* Corresponding author. Tel.: +90 232 7506789; fax: +90 2327506701.

E-mail address: tuhfegocmen@iyte.edu.tr (T. Göçmen).
}

edge noise, separation - stall noise, laminar boundary layer vortex shedding noise, trailing edge bluntness - vortex shedding noise and turbulent inflow noise [2]. Some early researchers were considering the tip vortex formation as the fifth noise mechanism [3].

Turbulent boundary layer trailing edge noise; is the noise that is occurred due to the formation of turbulence and wakes around the trailing edge of the airfoil. Separation - stall noise exists where the flow is separated around the airfoil. Laminar boundary layer vortex shedding noise occurs when a laminar boundary layer exists on any side of an airfoil. Trailing edge bluntness - vortex shedding noise is the noise that is formed due to the vortex shedding caused by the bluntness of the trailing edge. Turbulent inflow noise is due to the characteristics of the incoming flow.

The optimization process, which is based on the geometrical changes done on the airfoils, has been applied to profiles of FX 63137, S822, S834, SD2030, SG6043 and SH3055. They are chosen to be optimized since they may be considered to be in widely use in small scale wind turbines [4].

The optimization process starts with the prediction of noise generation and the results of such analysis can be found in the studies of Kim et al. [5] in which turbulent inflow and turbulent 


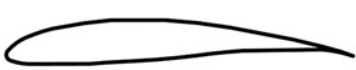

FX 63-137

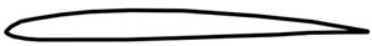

SD 2030

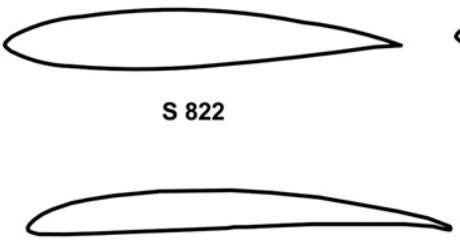

SG 6043

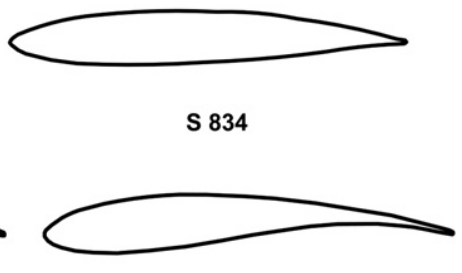

SH 3055

Fig. 1. The baseline airfoils

boundary layer trailing edge noises are analyzed and Son et al. [6] in which the calculations of self-noise are adjusted to the calculations of large range noise propagation with the terrain effects. Also, Jiang et al. [7] experimentally investigated the sound generation due to vortex shedding. In order to achieve airfoil geometry that emits lower levels of noise and yet aerodynamically sufficient enough, the algorithmic optimization methods have been used in the literature. Bertagnolio et al. [8] has considered the Trailing Edge Noise (TNO) model by Parchen et al. [9] and the model is put into the optimization program called "AIRFOILOPT" designed at Risø Laboratories. Marsden et al. [10] has applied the derivative-free optimization technique which minimizes the airfoil noise output due to vortex shedding. Another optimization model has been developed by Jouhaud et al. [11] and 2D Naca subsonic airfoil has been modified.

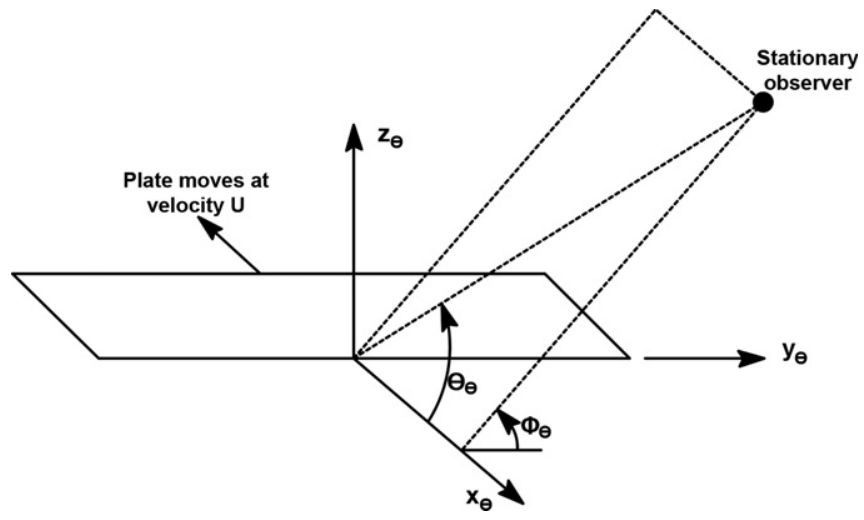

Fig. 2. Angles used in directivity functions.

Table 1

Turbine geometry factors and operating conditions.

\begin{tabular}{lr}
\hline Average wind speed $(\mathrm{m} / \mathrm{s})$ & 6 \\
Hub height $(\mathrm{m})$ & 25 \\
Blade radius $(\mathrm{m})$ & 7 \\
Rated power $(\mathrm{kW})$ & 10 \\
Number of blades & 3 \\
\hline
\end{tabular}

Airfoils in comparison :
Those methods are shown to be quite successful but require detailed study on the optimization techniques and computationally affordable for only two airfoil self-noise mechanisms at most. Therefore, more practical methods needed to be found. Bai et al. [12] placed a flow deflector on leading and trailing edges of S809 and FX60-100 airfoils and the flow separation is reduced, therefore
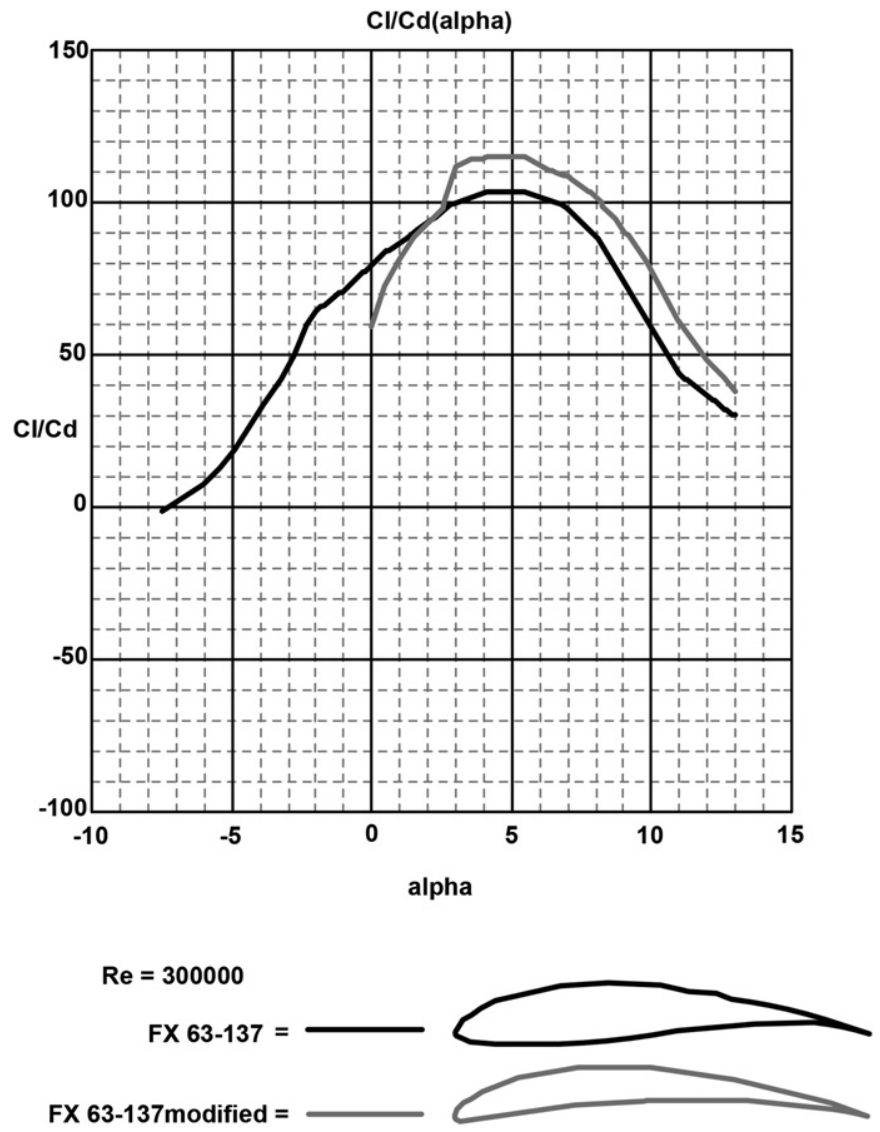

Fig. 4. Lift to drag ratios for FX 63-137 and modified.

FX $63-137=$

FX 63- 137 modified $=$

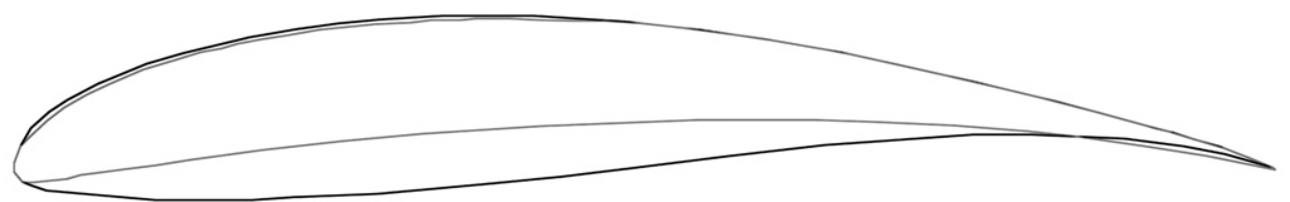

Fig. 3. Airfoil geometry comparison (FX 63-137 and modified). 


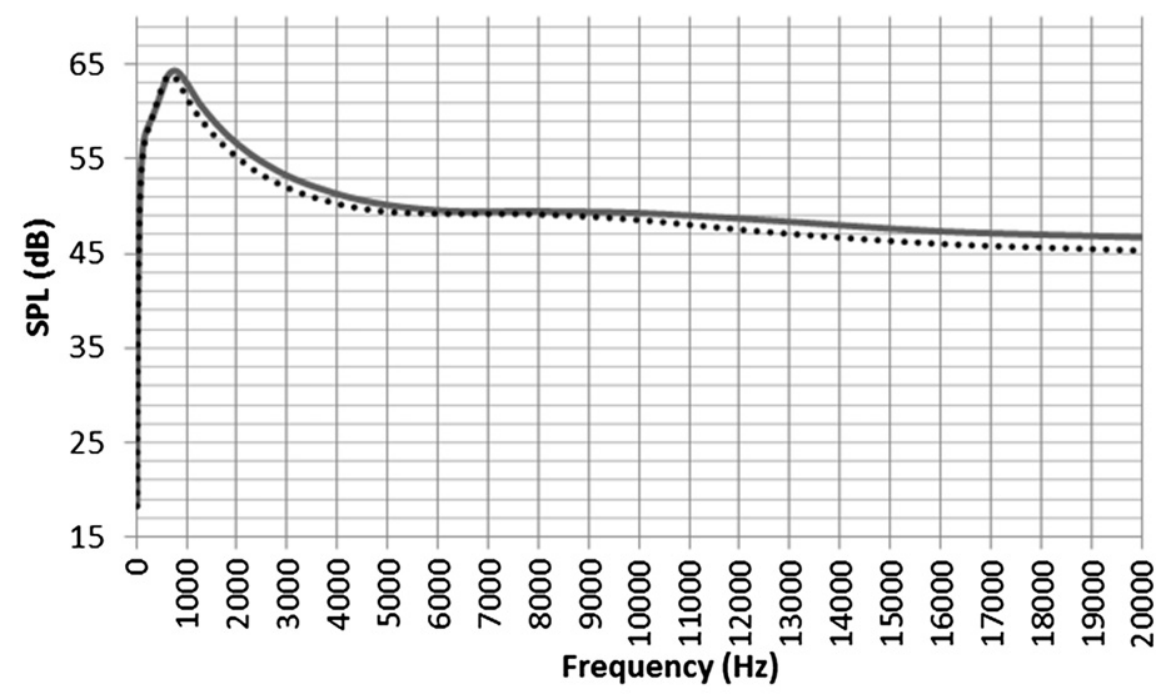

FX 63-137

\section{FX 63-137 modified}

Fig. 5. SPL spectra for FX 63-137 and modified.

noise is decreased where lift to drag ratio is increased. A more practical airfoil shape optimization has been done by decreasing the maximum thickness of the baseline airfoil basically [13]. This study is based on the geometrical approaches around the pressure side and the trailing edge of the profile. Those geometrical approaches include thinner trailing edge and engraved surface in the lower part of the airfoil and all five mechanisms of airfoil selfnoise have been taken into account. With this study, it was planned to reveal the basic geometrical specifications that an airfoil should have in order to reduce small scale wind turbine noise emission levels and six airfoils have been studied as examples. It is concluded that considered commercially available airfoils can be improved to emit lower self-noise and have better aerodynamic performances.

\section{Material and method}

The XFOIL code is used to predict the aerodynamic performances of the airfoils [14] which are shown in Fig. 1. The linear vorticity stream function panel method is used in this numerical approach and 2D flow field is constructed by three components: free stream flow, airfoil surface vortex sheet, and source sheet. The domain which is composed of airfoil contour and the wake is discretized into panels and each panel is assumed to have a linear vorticity profile and constant source strength. According to the study done by Hoogedoorn et al. [15], XFOIL has been validated especially for high Reynolds numbers which is the case of this study and is said to have capability to perform separation and stall calculations accurately.

While comparing the aerodynamic performances, according to the study edited by Spera et al. [16] the ratio of lift coefficient to drag coefficient is considered as the most important criterion.

The NAFNoise code [20] is used for the prediction of the airfoil self-noise and the numerical algorithm is based on the semiempirical models that are achieved by Brooks et al. [3], for the first four noise sources. For the last noise source that is listed as the turbulent inflow noise, the semi-empirical formula obtained by Amiet et al. [17] is taken into account. The validation of NAFNoise is studied by Moriarty et al. [18] for Mach number of 0.2 which is the case of this study.

\subsection{Semi-empirical noise models}

\subsubsection{Turbulent boundary layer trailing edge noise}

This is the first and perhaps the most common airfoil self - noise source especially for high Reynolds numbers. Based on the studies done by Brooks et al. [3] the turbulent boundary layer trailing edge noise along the pressure side of the airfoil is modeled as;

$\mathrm{SPL}_{p}=10 \log \left(\frac{\delta_{p}^{*} M^{5} L \bar{D}_{h}}{r_{e}^{2}}\right)+A\left(\frac{S t_{p}}{S t_{1}}\right)+\left(K_{1}-3\right)+\Delta K_{1}$

And for the suction side;

$\mathrm{S} 822=$

S822modified $=$

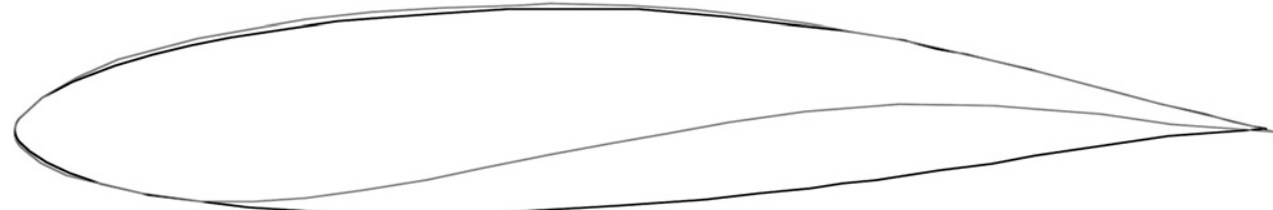

Fig. 6. Airfoil geometry comparison (S822 and modified). 


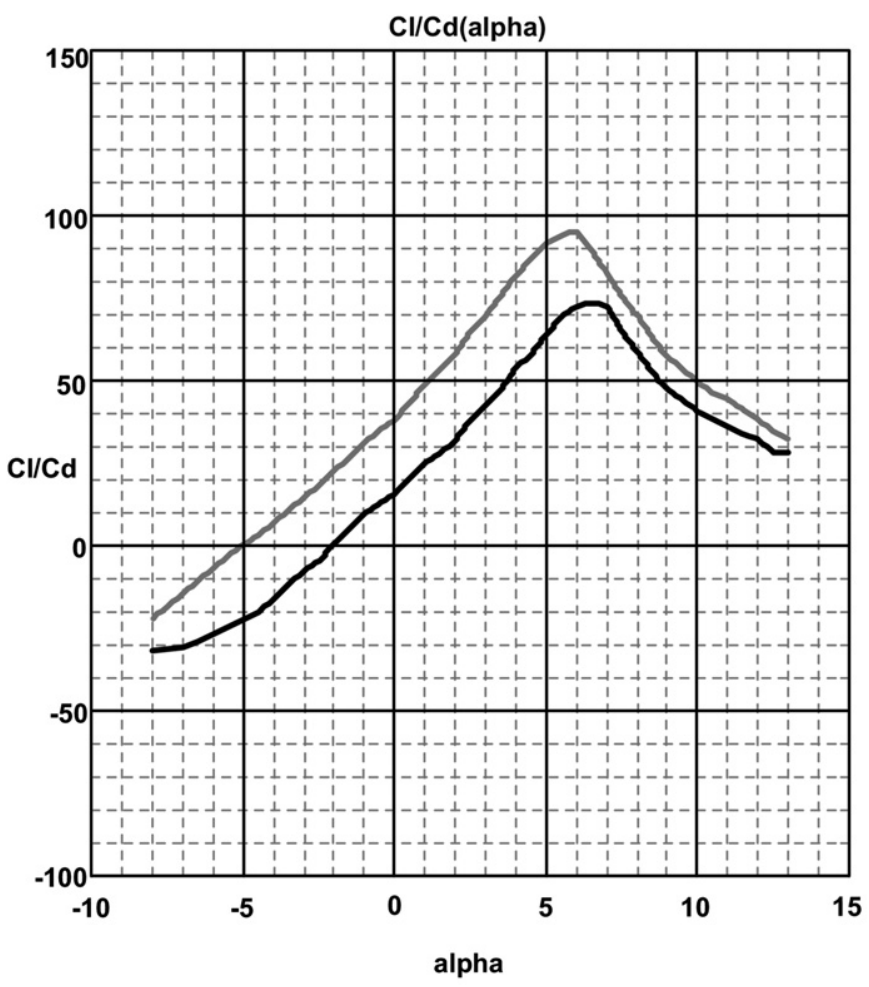

$\operatorname{Re}=\mathbf{3 0 0 0 0 0}$

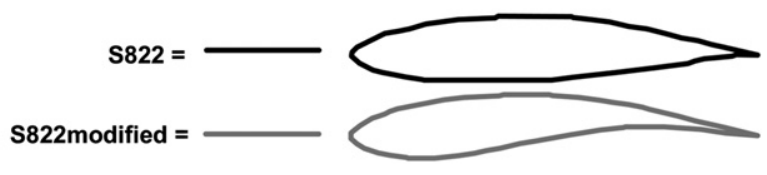

Fig. 7. Lift to drag ratios for S822 and modified.

$\mathrm{SPL}_{s}=10 \log \left(\frac{\delta_{s}^{*} M^{5} L \bar{D}_{h}}{r_{e}^{2}}\right)+A\left(\frac{S t_{s}}{S t_{1}}\right)+\left(K_{1}-3\right)$ where $\delta^{*}=\delta^{*}\left(\alpha, R e_{c}\right)$ is the boundary layer displacement thickness, based on $\alpha$, the angle of attack, and $\operatorname{Re}_{c}$, the Reynolds number based on chord. The subscript $p$ refers to the pressure side and $s$ to the suction side of the airfoil. Other parameters in Eqs. (1) and (2) are $L$, the span of the airfoil section; $r_{e}$, the effective observer distance; and $A$, an empirical spectral shape based on the Strouhal number, $S t=\left(f \delta^{*} / U\right)$, where $f$ is the frequency, and $U$ is the local mean velocity. Three other empirical relations are also used, $S t_{1}=0.02 M-0.6, K_{1}=K_{1}\left(R e_{c}\right)$, and $\Delta K_{1}=\Delta K_{1}\left(\alpha, R e_{\delta^{*}}\right)$. Also, $D_{h}$, the directivity function is defined in the equations since it is a correction factor considering the distance between source and the observer and therefore the Doppler effect and it may be described as [1];

$D_{h}\left(\Theta_{e} \Phi_{e}\right) \approx \frac{2 \sin ^{2}\left(1 / \Theta^{\Theta_{e}}\right) \sin ^{2} \Phi_{e}}{\left(1+M \cos \Theta_{e}\right)\left(1+\left(M-M_{C}\right) \cos \Theta_{e}\right)^{2}}$

where $M$ is the Mach number for airfoil section and $M_{c}$ is the convective Mach number and $\Theta_{e}$ and $\Phi_{e}$ are the directivity angles which are determined by Fig. 2 .

\subsubsection{Separation - stall noise}

For an airfoil, as the angle of attack, $\alpha$, increases within its boundaries, the separation around the suction side of the profile increases and that situation becomes more dominant on producing the noise. The semi - empirical formula of separation - stall noise, $\mathrm{SPL}_{\alpha}$ is very similar to Eq. (2) except that it has a remarkable dependency on $\alpha$.

The resulting scaling model for turbulent boundary layer trailing edge noise with noise due to separation and stall characteristics is then [1];

$\mathrm{SPL}_{\mathrm{TBL}-\mathrm{TE}}=10 \log \left(10^{\mathrm{SPL}_{p} / 10}+10^{\mathrm{SPL}_{\mathrm{S}} / 10}+10^{\mathrm{SPL}_{\alpha} / 10}\right)$

\subsubsection{Laminar boundary layer vortex shedding noise}

Since the characteristic of that type of noise is feedback amplification, it is tonal and therefore is not disturbing for larger scale wind turbines with higher Reynolds number. However, it is still a major concern for small scale ones which are the subject of this

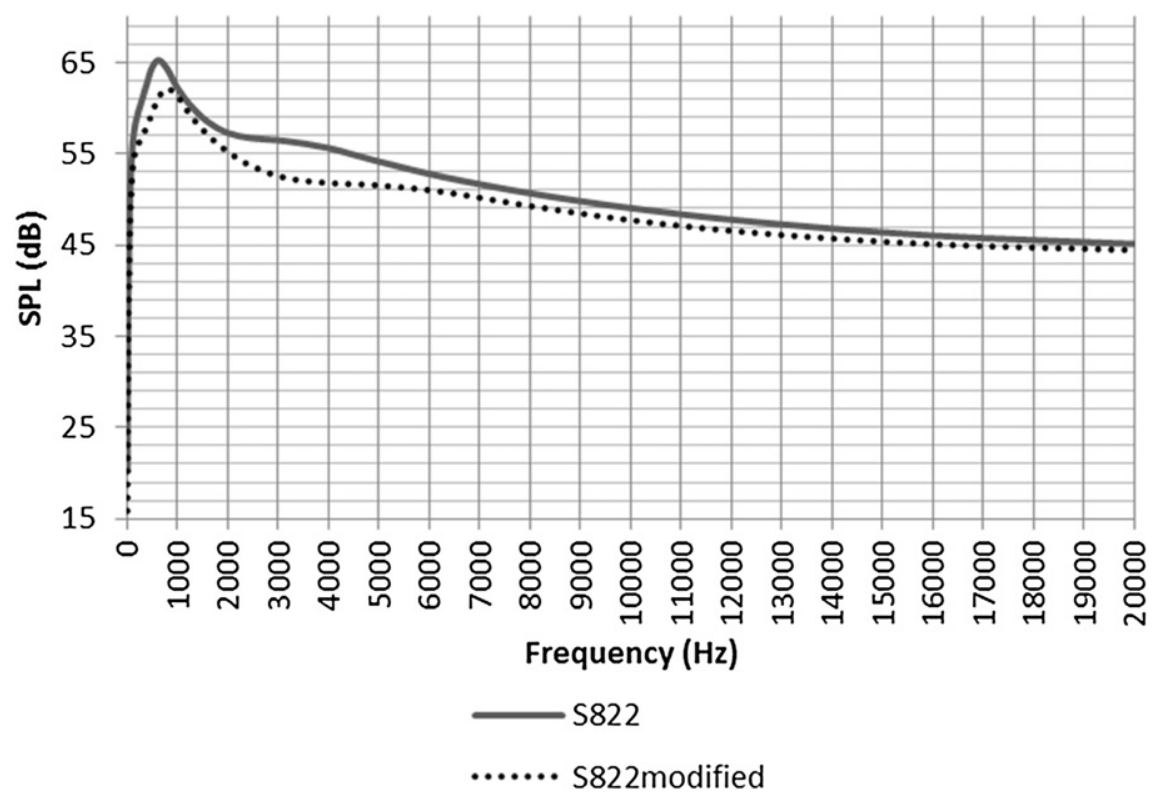

Fig. 8. SPL spectra for S822 and modified. 
S834modified $=$

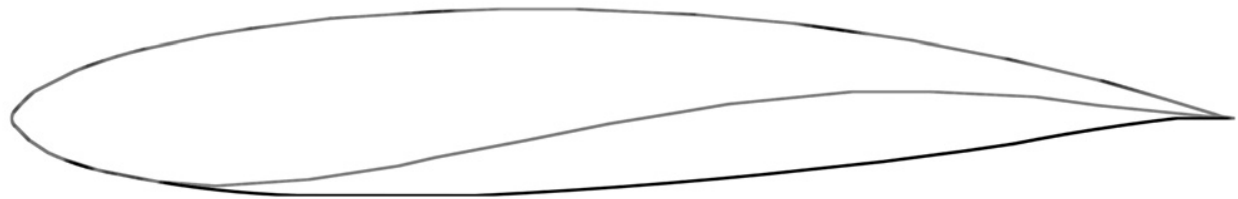

Fig. 9. Airfoil geometry comparison (S834 and modified).

study. The laminar boundary layer vortex shedding noise is described by Brooks et al. [3] as;

$$
\begin{aligned}
\mathrm{SPL}_{\mathrm{LBL}-\mathrm{VS}}= & 10 \log \left(\frac{\delta_{p}^{*} M^{5} L \bar{D}_{h}}{r_{e}^{2}}\right)+G_{1}\left(\frac{S t^{\prime}}{S t_{\text {peak }}^{\prime}}\right)+G_{2}\left(\frac{R e_{c}}{\left(R e_{c}\right)_{0}}\right) \\
& +G_{3}(\alpha)
\end{aligned}
$$

where most of the variables are already defined in Eq. (1) Other than those, which are $G_{1}, G_{2}$, and $G_{3}$ are empirical functions, $S t^{\prime}$ is the Strouhal number based on $\delta p, S t_{\text {peak }}^{\prime}=S t_{\text {peak }}^{\prime}\left(R e_{c}\right)$ and it is the peak Strouhal number and $\left(R e_{c}\right)_{o}$ is a reference Reynolds number depending on the angle of attack.

\subsubsection{Trailing edge bluntness - vortex shedding noise}

Since this source of noise emission from wind turbines is directly related with the airfoil geometry, the model includes some geometry-defined parameters. Trailing edge bluntness - vortex shedding noise is described as

$$
\begin{aligned}
\mathrm{SPL}_{\mathrm{TEB}-\mathrm{VS}}= & 10 \log \left(\frac{\delta_{p}^{*} M^{5} L \bar{D}_{h}}{r_{e}^{2}}\right)+G_{4}\left(\frac{h}{\delta_{\mathrm{avg}}^{*}}, \Psi\right) \\
& +G_{5}\left(\frac{h}{\delta_{\mathrm{avg}}^{*}}, \Psi, \frac{S t^{\prime \prime}}{S t_{\text {peak }}^{\prime \prime}}\right)
\end{aligned}
$$

where $h$ is the trailing edge thickness, $\delta_{\text {avg }}^{*}$ is the average displacement thickness for both sides of the airfoil, $\Psi$ is the solid angle between both airfoil surfaces just upstream of the trailing edge, $S t^{\prime \prime}$ is the Strouhal number based on $h, S t_{\text {peak }}^{\prime \prime}=S t_{\text {peak }}^{\prime \prime}\left(h / \delta_{\text {avg }}^{*}\right)$ is the peak Strouhal number; and $G_{4}$ and $G_{5}$ are empirical functions of these parameters.

\subsubsection{Turbulent inflow noise}

The incoming flow characteristics play an important role in this type of noise emission from the wind turbines. Different from the previous four models, this semi - empirical formula is studied by Amiet et al. [17].

$\mathrm{SPL}_{\text {Inflow }}=\mathrm{SPL}_{\text {Inflow }}^{H}+10 \log \left(\frac{\mathrm{LFC}}{1+\mathrm{LFC}}\right)$

$\mathrm{SPL}_{\text {Inflow }}^{H}=10 \log \left(\frac{\rho_{0}^{2} c_{0}^{2} l L}{2 r_{e}^{2}} M^{3} u^{2} I^{2} \frac{K^{3}}{\left(1+K^{2}\right)^{\frac{7}{3}}} \bar{D}_{L}\right)+58.4$

$\mathrm{LFC}=10 S^{2} M K^{2} \beta^{-2}$

$S^{2}=\left(\frac{2 \pi K}{\beta^{2}}+\left(1+2.4 \frac{K}{\beta^{2}}\right)^{-1}\right)^{-1}$

where $\rho_{o}$ is the air density, $c_{o}$ is the speed of sound, $l$ is a turbulence length scale, $u$ is the mean wind speed, $I$ is the turbulence intensity,
$K=\pi f c / U$, is the local wave number, where $f$ is the frequency of interest, $c$ is the local airfoil chord length, and $U$ is the local velocity over the airfoil section, $D_{L}$ is a low-frequency directivity function, LFC is a low - frequency correction factor, $S$ is the compressible Sears function; and $\beta^{2}=1-M^{2}$.

\section{Results and discussion}

During the optimization process, main idea was to change the shape of the pressure side of the airfoil since it is aerodynamically critical. Also the trailing edge of each airfoil has been well studied since most of the self-noise is originated from that location. Note that the calculations are done for a typical $10 \mathrm{~kW}$ wind turbine with a radius of $7 \mathrm{~m}$. The flow characteristics around the airfoils have been identified using the studies of Ünveren et al. [19] and the wind speed is averagely taken to be $6 \mathrm{~m} / \mathrm{s}$ for a hub height of $25 \mathrm{~m}$. The Reynolds number of that flow is calculated around $3.0 \times 10^{5}$ and Mach number calculation gives a value of 0.02 for an

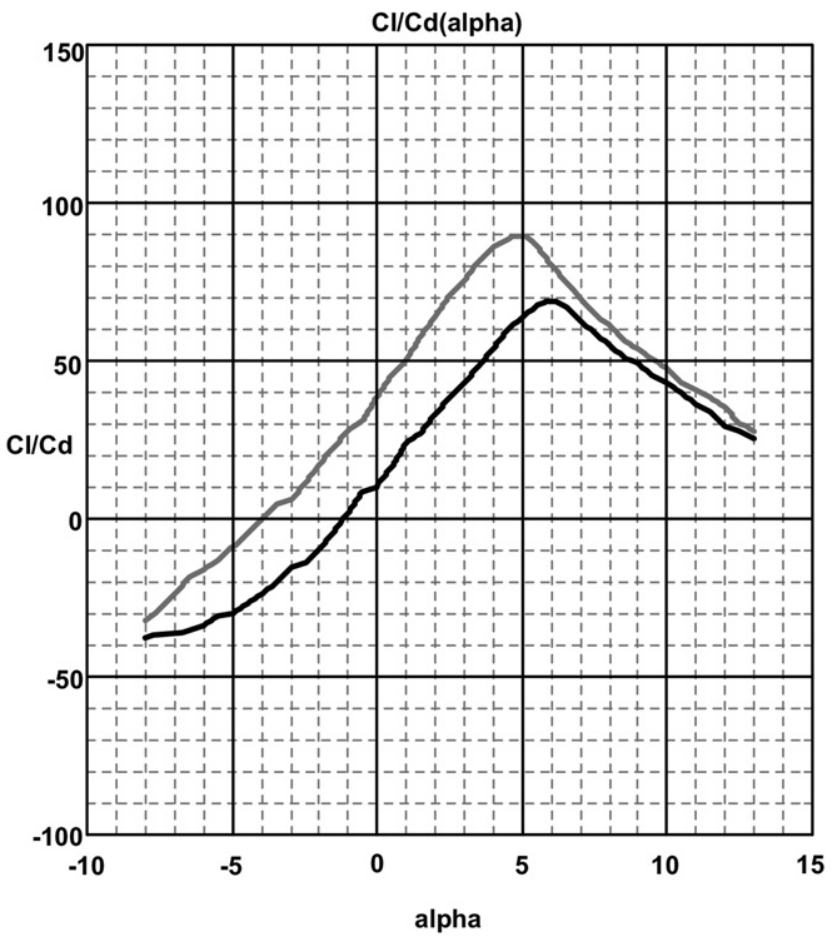

$\operatorname{Re}=300000$

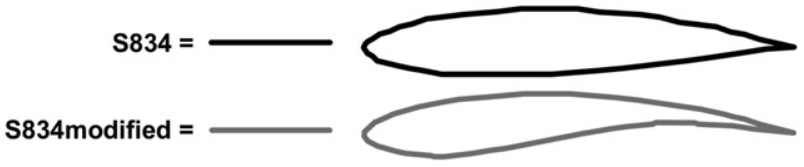

Fig. 10. Lift to drag ratios for S834 and modified. 


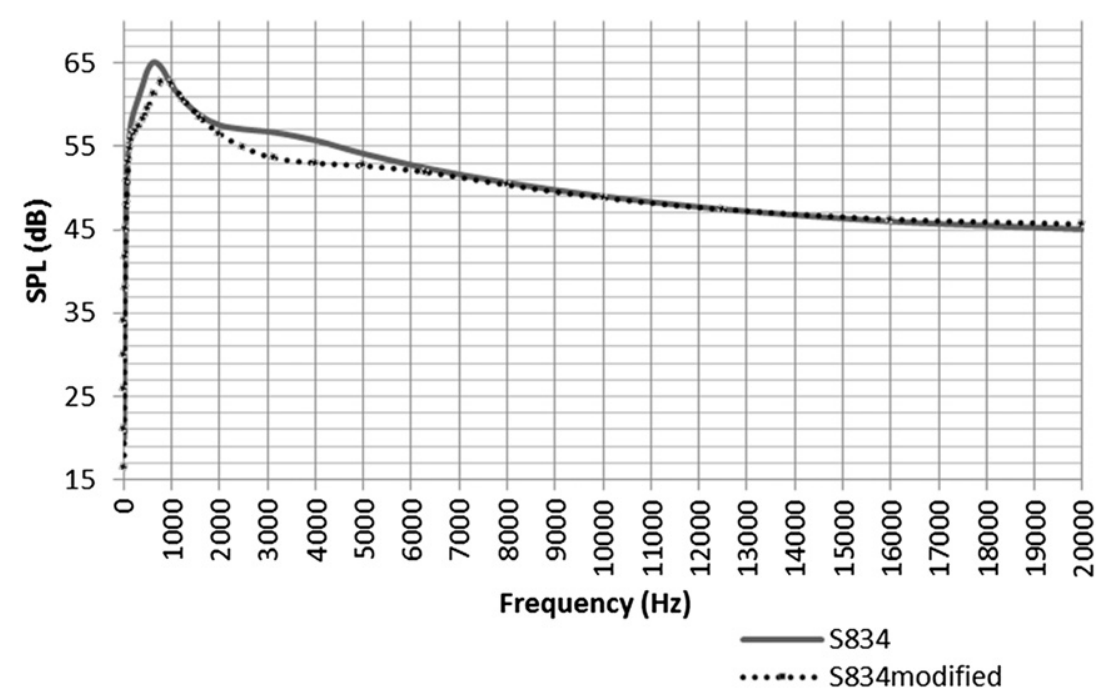

Fig. 11. SPL spectra for S834 and modified.

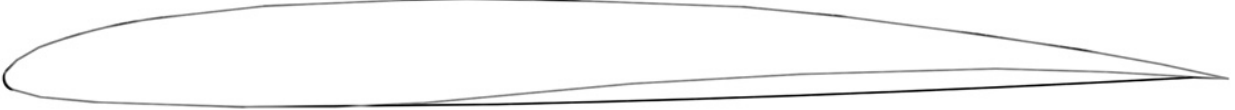

Fig. 12. Airfoil geometry comparison (SD2030 and modified).

incompressible flow. The results are satisfied with the desired aerodynamic performances (Table 1 ).

\subsection{Optimization of FX 63-137}

FX $63-137$ has maximum thickness of $13.71 \%$ at $30.9 \%$ of the chord and it has maximum camber of $5.97 \%$ at $53.3 \%$ of the chord and it is optimized to have $9.05 \%$ thick at $26.7 \%$ of the chord with a maximum camber of $7.75 \%$ at $43.6 \%$ at chord which is graphically shown in Fig. 3.

XFOIL code has been used to analyze and compare the most critical aerodynamic performance parameter which is the ratio of lift coefficient, $c_{L}$, and drag coefficient, $c_{D}$, with respect to the angle of attack, alpha and the outcome is shown graphically in Fig. 4.

As can be seen from Fig. 4, the lift to drag ratio of the modified version of FX 63-137 is more than 110 where for FX 63-137 that value is slightly above 100 .

The noise emission is compared by using the sound pressure level (SPL) spectra which is the outcome of the noise analysis tool, NAFNoise. For FX 63-137 airfoil optimization, the comparison between the SPL in $\mathrm{dB}$ is shown in Fig. 5.

The SPL spectra of FX 63-137 and FX 63-137modified show that the optimized version has a little lower levels of noise emission. Since the baseline profile is originally thin, the difference between the sound pressure levels is not that significant.

\subsection{Optimization of $S 822$}

The maximum thickness of that airfoil is $16.01 \%$ at $39.2 \%$ of the chord and it has maximum camber of $1.92 \%$ at $59.5 \%$ of the chord. The optimized geometry is $14.10 \%$ thick at $24.3 \%$ of the chord with

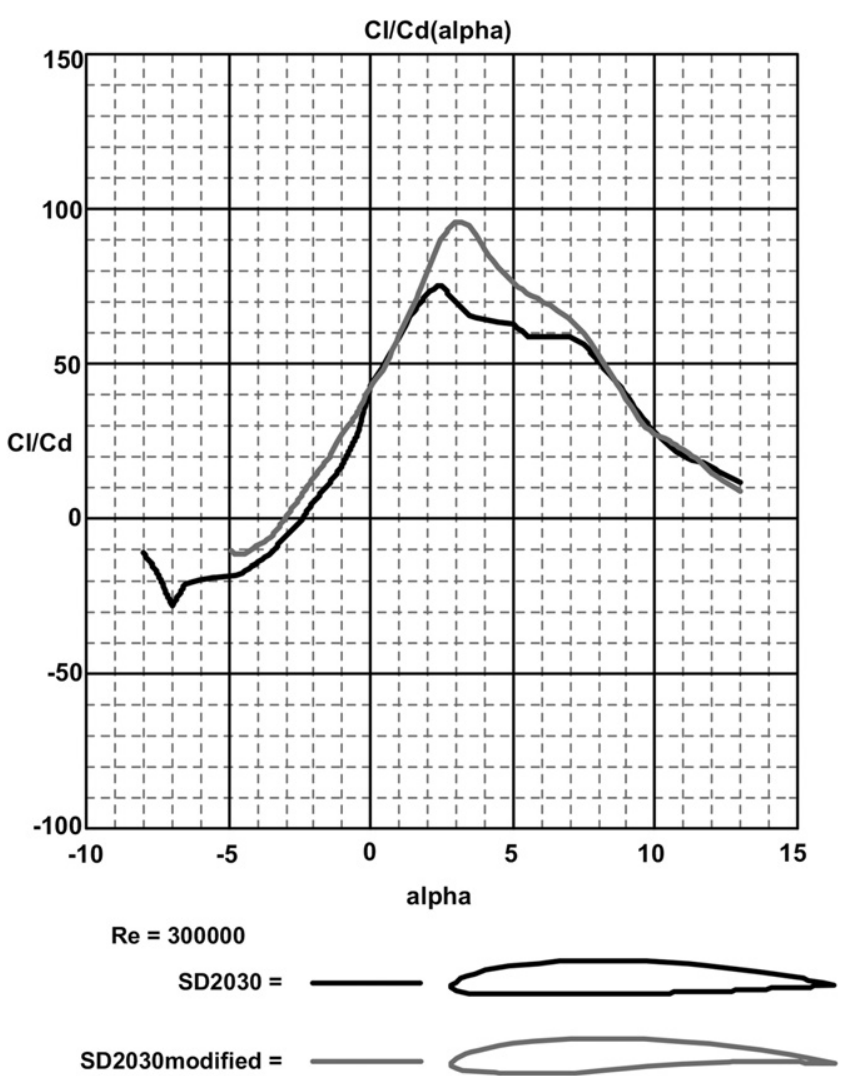

Fig. 13. Lift to drag ratios for SD2030 and modified. 


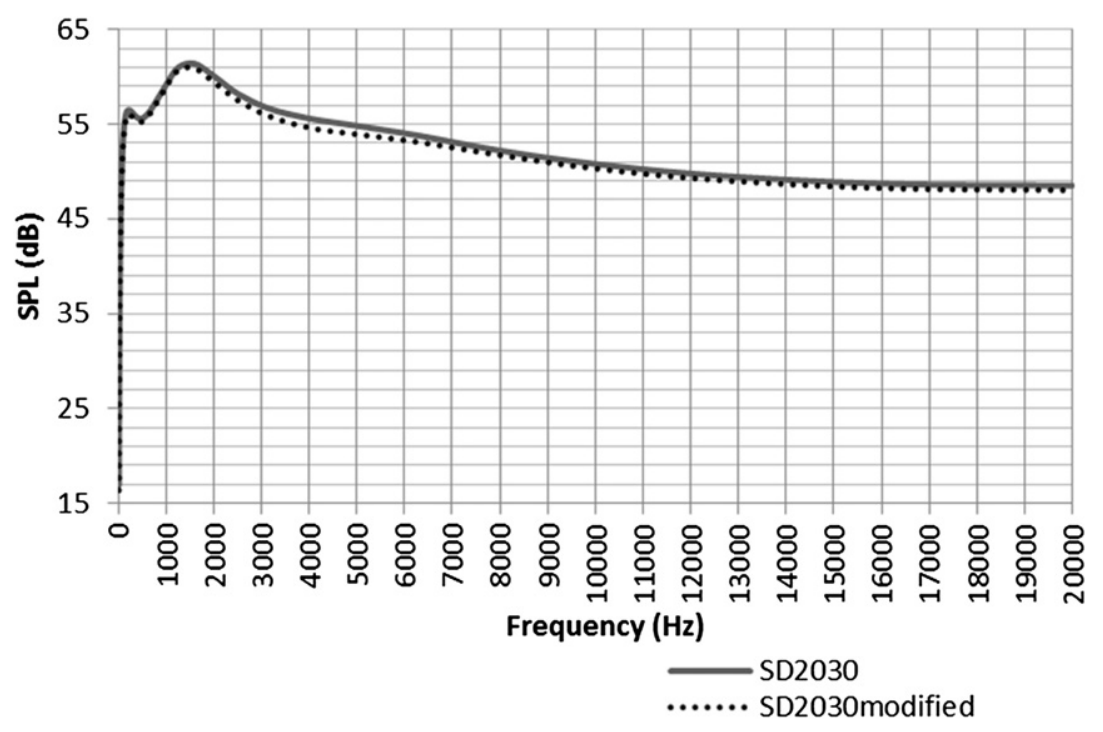

Fig. 14. SPL spectra for SD2030 and modified.

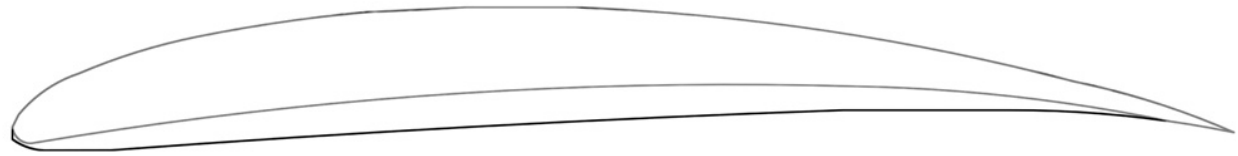

Fig. 15. Airfoil geometry comparison (SG6043 and modified).

a maximum camber of $5.15 \%$ at $61.0 \%$ of the chord. The geometry comparison between S822 and S822modified is shown in Fig. 6.

As can be seen from the Fig. 7, the pressure side of the baseline airfoil has been changed by increasing its curvature towards the trailing edge which is more effective in aerodynamics point of view. The comparison of performance obtained from the XFOIL tool is shown in Fig. 7.

Fig. 7 shows that, the lift to drag ratio has been increased from 70 to almost 95 with the help of the optimized geometry.

The noise emission levels are shown for S822 and its modified profile in Fig. 8.

The difference in the sound pressure levels of S822 and S822modified can be seen clearly in Fig. 8. Since the baseline profile is relatively thick, the geometry is easier to modify and the improvements in aerodynamics and noise emission level is significant.

\subsection{Optimization of $\mathrm{S} 834$}

S834 has $15.0 \%$ thickness at $39.5 \%$ at the chord and its maximum camber is $1.63 \%$ at $60.0 \%$ of the chord. The modified shape is $12.93 \%$ thick at $23.3 \%$ of the chord with a maximum camber of $4.69 \%$ at $63.1 \%$ of the chord. The comparison of those two geometries is shown in Fig. 9.

Using the same approach with the modification of S822, the pressure side of S834 is carved smoothly towards the trailing edge. The performance outcome of that geometric change may be seen in Fig. 10.

The maximum lift to drag ratio has been shifted from 70 to 90 which means a valuable progress in the performance of the airfoil in terms of wind turbine applications.

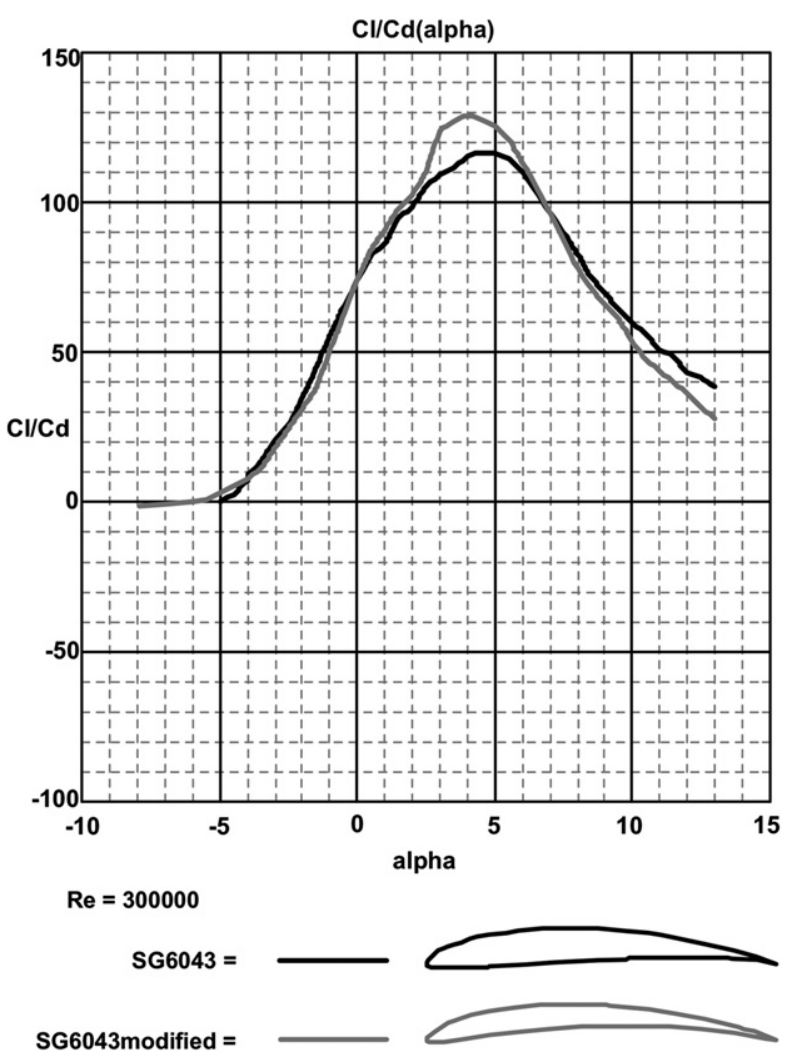

Fig. 16. Lift to drag ratios for SG6043 and modified. 


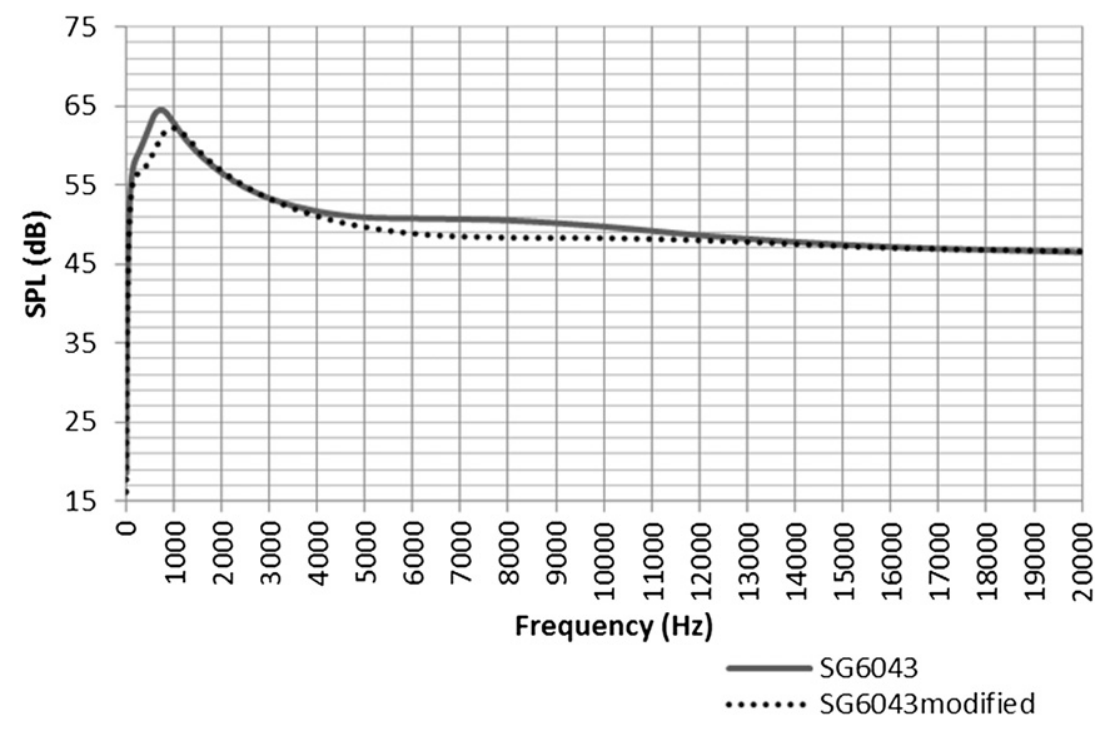

Fig. 17. SPL spectra for SG6043 and modified.

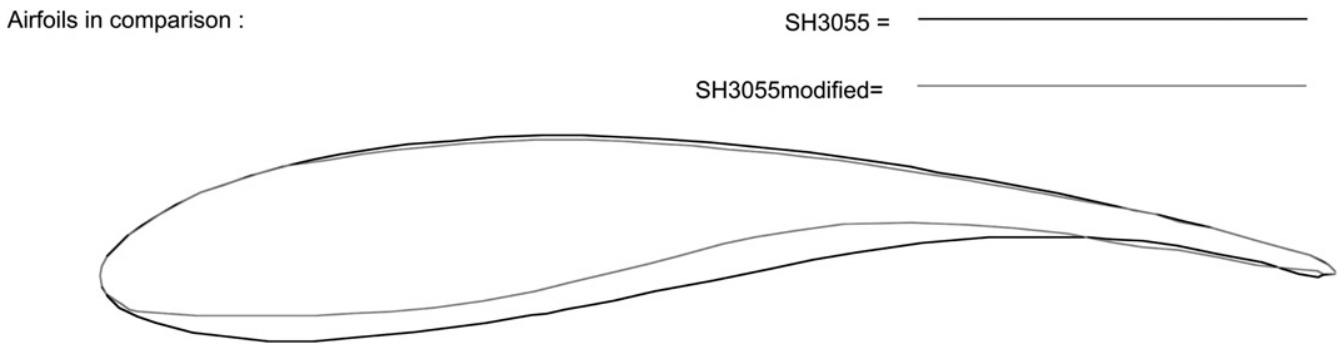

Fig. 18. Airfoil geometry comparison (SH3055 and modified).

The noise levels are compared in Fig. 11 which has been obtained by the tool of NAFNoise.

The sound pressure level spectra for S834 optimization shows that the noise emission level of modified shape is clearly reduced. It can also be interpreted from the Fig. 11 that the decrease is more significant in the frequency range of $50-2000 \mathrm{~Hz}$.

\subsection{Optimization of SD2030}

SD2030 is the baseline airfoil for this optimization and it has geometrical properties as; maximum thickness of $8.56 \%$ at $35.2 \%$ of the chord and maximum camber of $2.25 \%$ at $45.7 \%$ of the chord. The resulting optimized airfoil has; $8.52 \%$ thickness at $30.2 \%$ at the chord with a maximum camber of $2.94 \%$ at $54.0 \%$ of the chord. The geometries are shown in Fig. 12.

Since the airfoil is relatively thin, the effective way of modifying may be to change the camber characteristics and Fig. 12 shows the modification.

The aerodynamic performance comparison of those airfoils is given in Fig. 13.

Even though geometrically the difference is not that significant, since the pressure side of the baseline airfoil has been studied, the performance increased in certain extent. In Fig. 13, it can easily be seen that with the modification, not only the lift to drag ratio has been increased but also the distribution of that ratio becomes more stable with respect to angle of attack.

Since the baseline airfoil is thin, as mentioned, the difference not being significant was expected. However, the SPL spectra show in Fig. 14 that the decrease in the noise level is achieved especially around $4000-7000 \mathrm{~Hz}$ ranges.

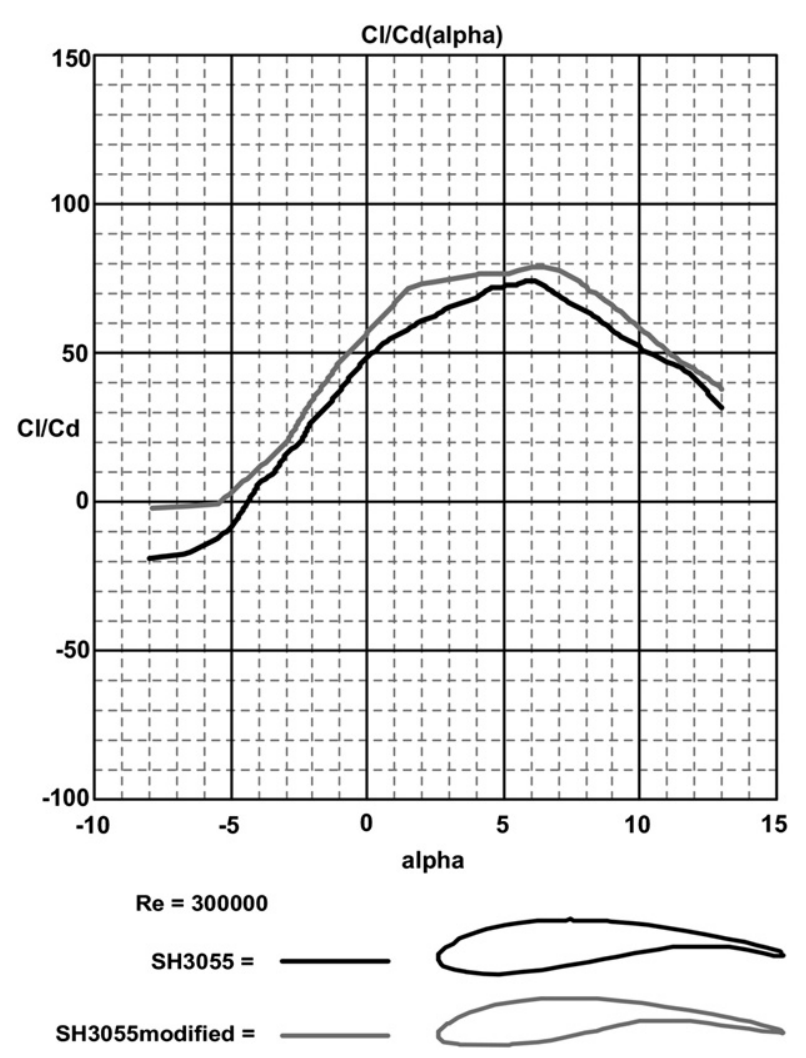

Fig. 19. Lift to drag ratios for SH3055 and modified. 


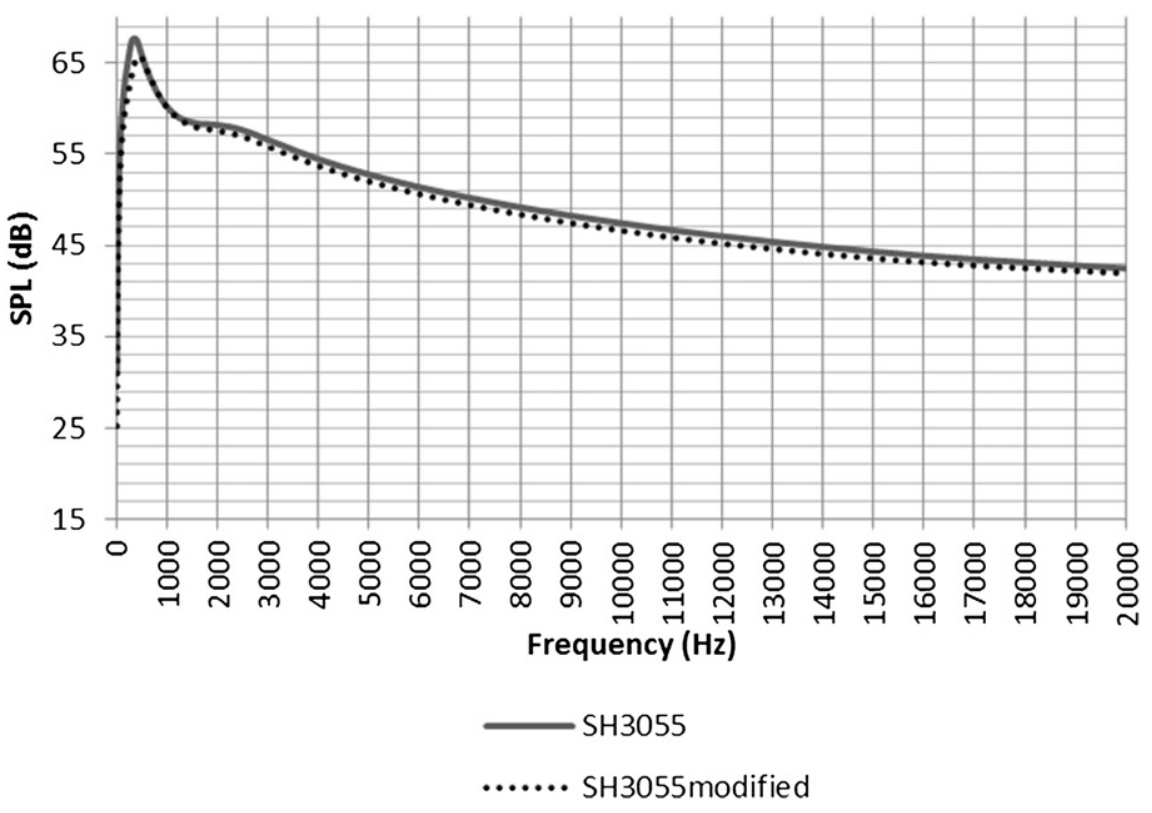

Fig. 20. SPL spectra for SH3055 and modified.

\subsection{Optimization of SG6043}

SG6043 is another relatively thin airfoil used in the small scale wind turbines with a thickness of $10.0 \%$ at $32.3 \%$ of the chord and maximum camber of $5.45 \%$ at $48.8 \%$ of the chord. SG6043modified which is the optimized airfoil has a maximum thickness of $7.13 \%$ at $25.9 \%$ of the chord and its camber is distributed with a maximum value of $6.98 \%$ at $46.6 \%$ of the chord. They are seen schematically in Fig. 15.

The optimization process of SG6043 includes the changes made in thickness and camber distribution, as can be seen in Fig. 15. The outcome of this optimized pressure side aerodynamically is shown in Fig. 16.

SG6043modified has higher maximum lift to drag ratio around its design angle of attack, $\alpha_{\text {design. }}$. Fig. 16 also shows that the values of $c_{L} / c_{D}$ of the optimized shape are below the ones that belong to the baseline airfoil. However, since corresponding angle of attacks are already in the region where stall occurs, the performance of the wind turbine will be at its minimum regardless to its lift to drag ratio. Thus, the optimization may still be considered satisfactory in terms of aerodynamic performance.

The airfoil self-noise characteristics of those two profiles are given in Fig. 17.

The noise emission levels differ tremendously especially for relatively lower frequencies because of the fact that the modified shape of SG6043 profile has smoother trailing edge and the overall thickness is lower.

\subsection{Optimization of $\mathrm{SH} 3055$}

SH3055 is one of the widely used airfoil in small scale wind turbine business and it is really successful in terms of consumer satisfaction. However, this optimization process shows that even this profile may be modified to have lower noise emission levels while its performance is increased.

The maximum thickness of $\mathrm{SH} 3055$ is $15.11 \%$ at $25.2 \%$ of the chord and the maximum camber of this profile is $5.40 \%$ at $62.9 \%$ of the chord. The modified version, SH3055 modified, is $13.14 \%$ thick at $26.5 \%$ of the chord where the maximum camber is $6.57 \%$ at $57.4 \%$ of the chord. The geometrical comparison of those two airfoils is included in Fig. 18.

As can be seen from Fig. 18, especially towards the leading edge, the overall thickness of the baseline airfoil is reduced. The reason for that is the trailing edge performance of SH3055 is satisfactory and no need for further improvements. However, by sharpening the nose section of the profile has improved the flow uniformity around the airfoil and therefore reduced the additional boundary layer formation which directly reduces noise and increases the performance.

The performance criteria of the airfoils mentioned above is compared in Fig. 19.

The $c_{L} / c_{D}$ distribution of SH3055 modified is not only higher but also more stable meaning that there is no significant peak points which is important for the turbine to perform at its best in various angles of attack.

As can be seen from Fig. 20, the noise emission level of SH3055 is also decreased quite significantly.

\section{Conclusion}

Airfoil low noise and high performance design optimization has been carried out for a typical three bladed, $10 \mathrm{~kW}$ wind turbine which has a radius of $7 \mathrm{~m}$. In this study, FX 63-137, S822, S834, SD2030, SG6043 and SH3055 which are the most widely used airfoils on the small scale wind turbine have been studied and each of them have been developed to have lower noise emission and higher aerodynamic performance, separately. In order to achieve that goal, the flow analysis tool XFOIL and airfoil self-noise analysis tool NAFNoise have been used. While changing the geometry of the airfoils, especially the pressure side and the trailing edge characteristics have been modified since they are the most critical locations particularly for aerodynamic and aero-acoustic concerns, respectively. In order to compare those two outcomes of the optimization, lift to drag criterion and SPL spectra has been drawn. The difference between the baseline and optimized versions of relatively thinner airfoils are not seen significantly because the geometrical changes made on those thin profiles are not allowed to be substantial. However, even for the thin profiles, the 
improvement in noise and performance characteristics is clearly shown. It can be seen that, with the geometrical optimization applied, the lift to drag ratios are increased whereas the noise emission levels are decreased up to $5 \mathrm{~dB}$. Each of the optimized airfoils has been shown to have better aerodynamic performance and lower noise emission than their baseline profiles.

\section{Nomenclature}

$\delta^{*} \quad$ boundary layer displacement thickness, $\mathrm{m}$

$\alpha$, alpha the angle of attack, deg

$\mathrm{Re}_{\mathrm{C}} \quad$ Reynolds number based on chord

$L \quad$ span of the airfoil section, $m$

$r_{e}$, effective observer distance, $m$

A empirical spectral shape based on the

St Strouhal number

$f \quad$ frequency, $\mathrm{Hz}$

$U \quad$ local mean velocity, $\mathrm{m} / \mathrm{s}$

$D_{h} \quad$ directivity function

$M \quad$ Mach number

$M_{c} \quad$ convective Mach number

$\Theta_{e}, \Phi_{e} \quad$ directivity angles

$\mathrm{SPL}_{\alpha} \quad$ separation - stall noise, $\mathrm{dB}$

$\mathrm{SPL}_{\mathrm{TBL}-\mathrm{TE}}$ turbulent boundary layer trailing edge noise, $\mathrm{dB}$

$\mathrm{SPL}_{p} \quad$ trailing edge noise along the pressure side of the airfoil

$\mathrm{SPL}_{S} \quad$ trailing edge noise along the suction side of the airfoil

SPL $L_{L B L}$-vs laminar boundary layer vortex shedding noise, $\mathrm{dB}$

$G_{1}, G_{2}, G_{3}$ empirical functions

$S t^{\prime} \quad$ Strouhal number based on $\delta p^{*}$

$S t_{\text {peak }}^{\prime} \quad$ peak Strouhal number

$\left(R e_{c}\right)_{0} \quad$ reference Reynolds number depending on the angle of attack

SPL $L_{\text {TEB-Vs }}$ trailing edge bluntness - vortex shedding noise, $d B$

$h$ trailing edge thickness, $m$

$\delta_{\mathrm{avg}}^{*} \quad$ average displacement thickness for both sides of the airfoil, $\mathrm{m}$

$\Psi \quad$ solid angle between both airfoil surfaces just upstream of the trailing edge, deg

$S t^{\prime \prime} \quad$ Strouhal number based on $h$

$S t_{\text {peak }}^{\prime \prime} \quad$ peak Strouhal number based on $h$

$G_{4}, G_{5}$ empirical functions

$\mathrm{SPL}_{\text {Inflow }}$ turbulent inflow noise, $\mathrm{dB}$

$\rho_{0} \quad$ air density, $\mathrm{kg} / \mathrm{m}^{3}$

$c_{0} \quad$ speed of sound, $\mathrm{m} / \mathrm{s}$

$l \quad$ turbulence length scale, $\mathrm{m}$

$u \quad$ mean wind speed, $\mathrm{m} / \mathrm{s}$

I turbulence intensity, \%

$K \quad$ local wave number
C local airfoil chord length, $m$

$D_{L} \quad$ low-frequency directivity function

LFC low-frequency correction factor

$S \quad$ compressible Sears function

\section{References}

[1] Wagner S, Bareiss R, Guidati G. Wind turbine noise. Germany: Springer Verlag; 1996.

[2] Leloudas G, JZhu W, Sørensen JN, Shen WZ, Hjort S. Prediction and reduction of noise for a $2.3 \mathrm{MW}$ wind Turbine, the Science of making torque from wind. Journal of Physics: Conference Series 2007;75:1-9.

[3] Brooks TF, Pope DS, Marcolini MA. Airfoil self-noise and prediction. NASA Ref. Pub; 1989. p. 1218.

[4] Migliore P, Oerlemans S. Wind tunnel aeroacoustic tests of six airfoils for use on small wind turbines. National Renewable Energy Laboratory; 2003.

[5] Kim H, Lee S, Son E, Lee S, Lee S. Aerodynamic noise analysis of large horizontal axis wind turbines considering fluid-structure interaction. Renewable Energy 2012;42:46-53.

[6] Son E, Kim H, Kim H, Choi W, Lee S. Integrated numerical method for the prediction of wind turbine noise and the long range propagation. Current Applied Physics 2010;10:S316-9.

[7] Jiang M, Li XD, Zhou JJ. Experimental and numerical investigation on sound generation from airfoil-flow interaction. Applied Mathematics and Mechanics-English Edition 2011;32(6):765-76.

[8] Bertagnolio F, Madsen HA, Bak C. Trailing edge noise model validation and application to airfoil optimization. Journal of Solar Energy-Transactions of ASME 2010;132(3)

[9] Parchen R. Progress report DRAW: a prediction scheme for trailing edge noise based on detailed boundary-layer characteristics. The Netherlands: TNO Institute of Applied Physics. Report No. HAG-RPT-980023, http://www.tno.nl/ index.cfm; 1998.

[10] Marsden AL, Wang M, Dennis JE, Moin P. Suppression of vortex-shedding noise via derivative-free shape optimization. Physics of Fluids 2004;16(10): L83-6.

[11] Jouhaud JC, Sagaut P, Montagnac M, Laurenceau J. A surrogate-model based multidisciplinary shape optimization method with application to a 2D subsonic airfoil. Computers and Fluids 2007;36(3):520-9.

[12] Bai YL, Ma XY, Ming X. Lift enhancement of airfoil and tip flow control for wind turbine. Applied Mathematics and Mechanics-English Edition 2011; 32(7):825-36.

[13] Kim T, Lee S, Kim H, Lee S. Design of low noise airfoil with high aerodynamic performance for use on small wind turbines. Science China 2010; 53:75-9.

[14] Drela M. XFOIL: an analysis and design system for low Reynolds number airfoils. In: Mueller TJ, editor. Low Reynolds numbers aerodynamics, lecture notes in engineering. New York: Springer; 1989. p. 54-61.

[15] Hoogendoorn E, Jacobs GB, Beyene A. Aero-elastic behavior of a flexible blade for wind turbine application: a 2D computational study. Energy 2010;35: 778-85.

[16] Spera DA. Wind turbine technology. 2nd ed. New York: ASME Press; 2009.

[17] Amiet RK. Acoustic radiation from an airfoil in a turbulent stream. Journal of Sound Vibration 1975;41(4):407-20.

[18] Moriarty P. NAFNoise user's guide; 2005. Golden Colorado.

[19] Ünveren C. Wind Resource Assessment on the Campus Area of Izmir Institute of Technology: Use of Multi Point Data Source MSc thesis, Izmir Institute of Technology, 2010.

[20] Moriarty P, Migliore P. Semi-empirical aeroacoustic noise prediction code for wind turbines. National Renewable Energy Laboratory; 2003. 